\title{
Asymptotic Behavior of Equilibrium Point for a Family of Rational Difference Equations
}

\author{
Chang-you Wang, ${ }^{1,2,3}$ Qi-hong Shi, ${ }^{4}$ and Shu Wang ${ }^{3}$ \\ ${ }^{1}$ College of Mathematics and Physics, Chongqing University of Posts and Telecommunications, \\ Chongqing 400065, China \\ ${ }^{2}$ Key Laboratory of Network Control and Intelligent Instrument, Chongqing University of \\ Posts and Telecommunications, Ministry of Education, Chongqing 400065, China \\ ${ }^{3}$ College of Applied Sciences, Beijing University of Technology, Beijing 100124, China \\ ${ }^{4}$ Fundamental Department, Hebei College of Finance, Baoding 071051, China
}

Correspondence should be addressed to Chang-you Wang, wangcy@cqupt.edu.cn

Received 7 August 2010; Accepted 19 October 2010

Academic Editor: Rigoberto Medina

Copyright (C) 2010 Chang-you Wang et al. This is an open access article distributed under the Creative Commons Attribution License, which permits unrestricted use, distribution, and reproduction in any medium, provided the original work is properly cited.

This paper is concerned with the following nonlinear difference equation $x_{n+1}=\sum_{i=1}^{l} A_{s_{i}} x_{n-s_{i}} /(B+$ $\left.C \prod_{j=1}^{k} x_{n-t_{j}}\right)+D x_{n}, n=0,1, \ldots$, where the initial data $x_{-m}, x_{-m+1}, \ldots, x_{-1}, x_{0} \in \mathbb{R}^{+}, m=$ $\max \left\{s_{1}, \ldots, s_{l}, t_{1}, \ldots, t_{k}\right\}, s_{1}, \ldots, s_{l}, t_{1}, \ldots, t_{k}$ are nonnegative integers, and $A_{s_{i}}, B, C$, and $D$ are arbitrary positive real numbers. We give sufficient conditions under which the unique equilibrium $\bar{x}=0$ of this equation is globally asymptotically stable, which extends and includes corresponding results obtained in the work of Çinar (2004), Yang et al. (2005), and Berenhaut et al. (2007). In addition, some numerical simulations are also shown to support our analytic results.

\section{Introduction}

Difference equations appear naturally as discrete analogues and in the numerical solutions of differential and delay differential equations, and they have applications in biology, ecology, physics, and so forth [1]. The study of properties of nonlinear difference equations has been an area of intense interest in recent years. There has been a lot of work concerning the globally asymptotic behavior of solutions of rational difference equations (e.g., see [2-5]). In particular, Çinar [6] studied the properties of positive solution to

$$
x_{n+1}=\frac{x_{n-1}}{1+x_{n} x_{n-1}}, \quad n=0,1, \ldots
$$


Yang et al. [7] investigated the qualitative behavior of the recursive sequence

$$
x_{n+1}=\frac{a x_{n-1}+b x_{n-2}}{c+d x_{n-1} x_{n-2}}, \quad n=0,1, \ldots
$$

More recently, Berenhaut et al. [8] generalized the result reported in [7] to

$$
x_{n}=\frac{y_{n-k}+y_{n-m}}{1+y_{n-k} y_{n-m}}, \quad n=0,1, \ldots
$$

For more similar work, one can refer to [9-14] and references therein.

The main theorem in this paper is motivated by the above studies. The essential problem we consider in this paper is the asymptotic behavior of the solutions for

$$
x_{n+1}=\frac{\sum_{i=1}^{l} A_{s_{i}} x_{n-s_{i}}}{B+C \prod_{j=1}^{k} x_{n-t_{j}}}+D x_{n}, \quad n=0,1, \ldots
$$

with initial data $x_{-m}, x_{-m+1}, \ldots, x_{-1}, x_{0} \in \mathbb{R}^{+}, m=\max \left\{s_{1}, \ldots, s_{l}, t_{1}, \ldots, t_{k}\right\}, s_{1}, \ldots, s_{l}, t_{1}, \ldots, t_{k}$ are nonnegative integers, and $A_{s_{i}}, B, C$, and $D$ are arbitrary positive real numbers. In addition, some numerical simulations of the behavior are shown to illustrate our analytic results.

This paper proceeds as follows. In Section 2, we introduce some definitions and preliminary results. The main results and their proofs are given in Section 3. Finally, some numerical simulations are shown to support theoretical analysis.

\section{Preliminaries and Notations}

In this section, we prepare some materials used throughout this paper, namely, notations, the basic definitions, and preliminary results. We refer to the monographs of Kocić and Ladas [2], and Kulenović and Ladas [3].

Let $\sigma$ and $\kappa$ be two nonnegative integers such that $\sigma+\kappa=n$. We usually write a vector $\mathbf{x}=\left(x_{1}, x_{2}, \ldots, x_{n}\right)$ with $n$ components into $\mathbf{x}=\left([\mathbf{x}]_{\sigma},[\mathbf{x}]_{\kappa}\right)$, where $[\mathbf{x}]_{\sigma}$ denotes a vector with $\sigma$-components of $\mathbf{x}$.

Lemma 2.1. Let I be some interval of real numbers and

$$
f: I^{k+1} \longrightarrow I
$$

be a continuously differentiable function. Then, for every set of initial conditions $x_{-k}, x_{-k+1}, \ldots, x_{0} \in$ $I$,

$$
x_{n+1}=f\left(x_{n}, x_{n-1}, \ldots, x_{n-k}\right), \quad n=0,1, \ldots
$$

has a unique solution $\left\{x_{n}\right\}_{n=-k}^{+\infty}$. 
Definition 2.2. Function $f: \mathbb{R}^{m} \rightarrow \mathbb{R}$ is called mixed monotone in subset $I^{m}$ of $\mathbb{R}^{m}$ if $f\left([\mathbf{x}]_{\sigma},[\mathbf{x}]_{\kappa}\right)$ is monotone nondecreasing in each component of $[\mathbf{x}]_{\sigma}$ and is monotone nonincreasing in every component of $[\mathbf{x}]_{\kappa}$ for $\mathbf{x} \in I^{m}$.

Definition 2.3. If there exists a point $\bar{x} \in I$ such that $\bar{x}=f(\bar{x}, \bar{x}, \ldots, \bar{x}, \bar{x}), \bar{x}$ is called an equilibrium point of (2.2).

Definition 2.4. Let $\bar{x}$ be an equilibrium point of (2.2).

(1) The equilibrium $\bar{x}$ of (2.2) is (locally) stable if for every $\varepsilon>0$, there exists $\delta>0$ such that for any initial data $\left(x_{-m}, x_{-m+1}, \ldots, x_{-1}, x_{0}\right) \in I^{m+1}$ satisfying $\max \left\{\mid x_{-m}-\right.$ $\left.\bar{x}|,| x_{-m+1}-\bar{x}|, \ldots,| x_{0}-\bar{x} \mid\right\}<\delta,\left|x_{n}-\bar{x}\right|<\varepsilon$ holds for all $n \geq-m$.

(2) The equilibrium $\bar{x}$ of (2.2) is a local attractor if there exists $\delta>0$ such that $\lim _{n \rightarrow \infty} x_{n}=\bar{x}$ for any data $\left(x_{-m}, x_{-m+1}, \ldots, x_{-1}, x_{0}\right) \in I^{m+1}$ satisfying $\max \left\{\mid x_{-m}-\right.$ $\left.\bar{x}|,| x_{-m+1}-\bar{x}|, \ldots,| x_{0}-\bar{x} \mid\right\}<\delta$.

(3) The equilibrium $\bar{x}$ of (2.2) is locally asymptotically stable if it is stable and is a local attractor.

(4) The equilibrium $\bar{x}$ of (2.2) is a global attractor if for all $x_{-m}, x_{-m+1}, \ldots, x_{-1}, x_{0} \in I$, $\lim _{n \rightarrow \infty} x_{n}=\bar{x}$ holds.

(5) $\bar{x}$ is globally asymptotically stable if it is stable and is a global attractor.

(6) $\bar{x}$ is unstable if it is not locally stable.

Lemma 2.5. Assume that $s_{1}, s_{2}, \ldots, s_{k} \in \mathbb{R}$ and $k \in N$. Then,

$$
\left|s_{1}\right|+\left|s_{2}\right|+\cdots+\left|s_{k}\right|<1
$$

is a sufficient condition for the local stability of the difference equation

$$
x_{n+k}+s_{1} x_{n+k-1}+\cdots+s_{k} x_{n}=0, \quad n=0,1, \ldots
$$

\section{The Main Results and Their Proofs}

In this section, we investigate the globally asymptotic stability of the equilibrium point of (1.4).

It is obvious that $\bar{x}=0$ is a unique equilibrium point of (1.4) provided either $0<D<$ $1, \sum_{i=1}^{l} A_{s_{i}}<B(1-D)$ or $D \geq 1$.

Let $f:\left(\mathbb{R}^{+}\right)^{m} \rightarrow \mathbb{R}^{+}$be a multivariate continuous function defined by

$$
f\left(x_{n-s_{1}}, \ldots, x_{n-s_{l}}, x_{n-t_{1}}, \ldots, x_{n-t_{k}}, x_{n}\right)=\frac{\sum_{i=1}^{l} A_{s_{i}} x_{n-s_{i}}}{B+C \prod_{j=1}^{k} x_{n-t_{j}}}+D x_{n}
$$


If $s_{1} \neq \cdots \neq s_{l} \neq t_{1} \neq \cdots \neq t_{k}$, we have

$$
\begin{aligned}
& f_{x_{n-s_{i}}}\left(x_{n-s_{1}}, \ldots, x_{n-s_{l}}, x_{n-t_{1}}, \ldots, x_{n-t_{k}}, x_{n}\right)=\frac{A_{s_{i}}}{B+C \prod_{j=1}^{k} x_{n-t_{j}}}, \quad s_{i} \neq 0, i=1,2, \ldots, l, \\
& f_{x_{n-s_{i}}}\left(x_{n-s_{1}}, \ldots, x_{n-s_{l}}, x_{n-t_{1}}, \ldots, x_{n-t_{k}}, x_{n}\right)=\frac{A_{s_{i}}}{B+C \prod_{j=1}^{k} x_{n-t_{j}}}+D, \quad \text { for some } s_{i}=0 \text {, } \\
& i \in\{1,2, \ldots, l\}, \\
& f_{x_{n-t_{j}}}\left(x_{n-s_{1}}, \ldots, x_{n-s_{l}}, x_{n-t_{1}}, \ldots, x_{n-t_{k}}, x_{n}\right)=\frac{-C \sum_{i=1}^{l} A_{s_{i}} x_{n-s_{i}}}{\left[B+C \prod_{r=1}^{k} x_{n-t_{r}}\right]^{2}} \prod_{r=1, r \neq j}^{k} x_{n-t_{r}}, \\
& t_{j} \neq 0, \quad j=1,2, \ldots, k, \\
& f_{x_{n-t_{j}}}\left(x_{n-s_{1}}, \ldots, x_{n-s_{l}}, x_{n-t_{1}}, \ldots, x_{n-t_{k}}, x_{n}\right)=\frac{-C \sum_{i=1}^{l} A_{s_{i}} x_{n-s_{i}}}{\left[B+C \prod_{r=1}^{k} x_{n-t_{r}}\right]^{2}} \prod_{r=1, r \neq j}^{k} x_{n-t_{r}}+D \text {, } \\
& \text { for some } t_{j}=0, \quad j \in\{1,2, \ldots, k\} \text {, } \\
& f_{x_{n}}\left(x_{n-s_{1}}, \ldots, x_{n-s_{l}}, x_{n-t_{1}}, \ldots, x_{n-t_{k}}, x_{n}\right)=D, \quad s_{i}, t_{j} \neq 0, \quad i=1,2, \ldots, l, \quad j=1,2, \ldots k \text {. }
\end{aligned}
$$

By constructing (1.4) and applying Lemma 2.5, we have the following affirmation.

Theorem 3.1. If $s_{1} \neq \cdots \neq s_{l} \neq t_{1} \neq \cdots \neq t_{k}$, and $\sum_{i=1}^{l} A_{s_{i}}<B(1-D)$ with $0<D<1$, then the unique equilibrium point $\bar{x}=0$ of (1.4) is locally stable. If $D \geq 1, \bar{x}=0$ is unstable.

Proof. Considering the linearized equation of (1.4) with respect to equilibrium point $\bar{x}=0$,

$$
\begin{gathered}
z_{n+1}+D z_{n}+\frac{\sum_{i=1}^{l} A_{s_{i}} z_{n-s_{i}}}{B}=0, \quad \text { for } s_{i} \neq 0, i=1,2, \ldots, l, \\
z_{n+1}+D z_{n}+\frac{\sum_{i=1}^{l} A_{s_{i}} z_{n-s_{i}}}{B}=0, \quad \text { for some } t_{j}=0, j \in\{1,2, \ldots, k\}, \\
z_{n+1}+D z_{n}+\frac{\sum_{i=1}^{l} A_{s_{i}} z_{n-s_{i}}}{B}=0, \text { for } s_{i}, \quad t_{j} \neq 0, i=1,2, \ldots, l, j=1,2, \ldots k, \\
z_{n+1}+\left(D+\frac{A_{s_{p}}}{B}\right) z_{n}+\frac{\sum_{i=1, i \neq p}^{l} A_{s_{i}} z_{n-s_{i}}}{B}=0, \text { for some } s_{p}=0, p \in\{1,2, \ldots, l\} .
\end{gathered}
$$

By Lemma 2.5, (1.4) is stable if the following inequality holds

$$
D+\frac{\sum_{i=1}^{l} A_{s_{i}}}{B}<1
$$

which implies our claim. 
When $D \geq 1$, note that the solution of (1.4) is $x_{n}>0$, hence $x_{n+1} \geq D x_{n} \geq x_{n}$, which implies that $\left\{x_{n}\right\}_{n=1}^{\infty}$ is a dispersed sequence.

Theorem 3.2. Let $f: \mathbb{R}^{m+1} \rightarrow \mathbb{R}$ defined by (2.2) be a continuous function satisfying the mixed monotone property. If there exists two real numbers $\Omega^{0}, \Theta^{0}$ satisfying

$$
\Omega^{0} \leq \min \left\{x_{-m}, x_{-m+1}, \ldots, x_{-1}, x_{0}\right\} \leq \max \left\{x_{-m}, x_{-m+1}, \ldots, x_{-1}, x_{0}\right\} \leq \Theta^{0},
$$

such that

$$
\Omega^{0} \leq f\left(\left[\Omega^{0}\right]_{\sigma^{\prime}}\left[\Theta^{0}\right]_{\kappa}\right) \leq f\left(\left[\Theta^{0}\right]_{\sigma^{\prime}}\left[\Omega^{0}\right]_{\kappa}\right) \leq \Theta^{0},
$$

then there exists $(\Omega, \Theta) \in\left[\Omega^{0}, \Theta^{0}\right]^{2}$ satisfying

$$
\Omega=f\left([\Omega]_{\sigma},[\Theta]_{\kappa}\right), \quad \Theta=f\left([\Theta]_{\sigma},[\Omega]_{\mathcal{K}}\right) .
$$

Moreover, if $\Omega=\Theta$, then (2.2) has a unique equilibrium point $\bar{x} \in\left[\Omega^{0}, \Theta^{0}\right]$, and every solution of (2.2) converges to $\bar{x}$.

Proof. Let $\Omega^{0}$ and $\Theta^{0}$ be a couple of initial iteration data, then we construct two sequences $\left\{\Omega^{i}\right\}_{i=1}^{\infty}$ and $\left\{\Theta^{i}\right\}_{i=1}^{\infty}$ in the form

$$
\Omega^{i}=f\left(\left[\Omega^{i-1}\right]_{\sigma^{\prime}}\left[\Theta^{i-1}\right]_{\kappa}\right), \quad \Theta^{i}=f\left(\left[\Theta^{i-1}\right]_{\sigma^{\prime}}\left[\Omega^{i-1}\right]_{\kappa}\right) .
$$

Note that mixed monotone property of $f$ and initial assumption, the sequences $\left\{\Omega^{i}\right\}_{i=1}^{\infty}$ and $\left\{\Theta^{i}\right\}_{i=1}^{\infty}$ thus possess

$$
\Omega^{0} \leq \Omega^{1} \leq \cdots \leq \Omega^{i} \leq \Theta^{i} \leq \cdots \leq \Theta^{1} \leq \Theta^{0}, \quad i=0,1,2, \ldots
$$

It guarantees one can choose a new sequence $\left\{x_{l}\right\}_{l=1}^{\infty}$ satisfying $\Omega^{i} \leq x_{l} \leq \Theta^{i}$ for $l \geq(m+1) i+1$. Denote

$$
\Omega=\lim _{i \rightarrow \infty} \Omega^{i}, \quad \Theta=\lim _{i \rightarrow \infty} \Theta^{i},
$$

then

$$
\Omega \leq \lim _{i \rightarrow \infty} \inf x_{i} \leq \lim _{i \rightarrow \infty} \sup x_{i} \leq \Theta
$$

By the continuity of $f$, we have

$$
\Omega=f\left([\Omega]_{\sigma},[\Theta]_{\kappa}\right), \quad \Theta=f\left([\Theta]_{\sigma},[\Omega]_{\kappa}\right) .
$$

Moreover, if $\Omega=\Theta$, then $\Omega=\Theta=\lim _{i \rightarrow \infty} x_{i}=\bar{x}$, and then the proof is complete. 
Theorem 3.3. If $0<D<1, \sum_{i=1}^{l} A_{s_{i}}<B^{2}(1-D) /((k+1) C+B)$, and $t_{j} \neq 0(j=1,2, \ldots, k)$, then the unique equilibrium point $\bar{x}=0$ of (1.4) is global attractor for any initial conditions $\left(x_{-m}, x_{-m+1}, \ldots, x_{1}, x_{0}\right) \in(0,1)^{m+1}$.

Proof. For any nonnegative initial data $x_{-i}, i \in\{0,1,2, \ldots, m\}$, it is obvious that the function $f\left(x_{n-s_{1}}, \ldots, x_{n-s_{l}}, x_{n-t_{1}}, \ldots, x_{n-t_{k}}, x_{n}\right)$ defined by (3.1) is nondecreasing in $\left(x_{n-s_{1}}, \ldots, x_{n-s_{l}}, x_{n}\right)$ and nonincreasing in $\left(x_{n-t_{1}}, \ldots, x_{n-t_{k}}\right)$.

Let

$$
\Omega^{0}=0, \quad \Theta^{0}=\max \left\{x_{-m}, \ldots, x_{-1}, x_{0}\right\}
$$

by view of the assumption $\sum_{i=1}^{l} A_{s_{i}}<B^{2}(1-D) /((k+1) C+B)$, we have

$$
\begin{aligned}
\Omega^{0} & \leq f\left(\left[\Omega^{0}\right]_{\sigma^{\prime}}\left[\Theta^{0}\right]_{k}\right)=\frac{\sum_{i=1}^{l} A_{s_{i}} \Omega^{0}}{B+C\left(\Theta^{0}\right)^{k}}+D \Omega^{0} \\
& \leq f\left(\left[\Theta^{0}\right]_{\sigma^{\prime}}\left[\Omega^{0}\right]_{\kappa}\right)=\frac{\sum_{i=1}^{l} A_{s_{i}} \Theta^{0}}{B+C\left(\Omega^{0}\right)^{k}}+D \Theta^{0} \\
& \leq \frac{\Theta^{0} \sum_{i=1}^{l} A_{s_{i}}}{B}+D \Theta^{0} \\
& \leq \frac{\Theta^{0}[(k+1) C+B] \sum_{i=1}^{l} A_{s_{i}}}{B^{2}}+D \Theta^{0} \leq \Theta^{0} .
\end{aligned}
$$

From (1.4) and Theorem 3.2, there exists $(\Omega, \Theta) \in\left[\Omega^{0}, \Theta^{0}\right]^{2}$ satisfying

$$
\Omega=\frac{\sum_{i=1}^{l} A_{s_{i}} \Omega}{B+C \Theta^{k}}+D \Omega, \quad \Theta=\frac{\sum_{i=1}^{l} A_{s_{i}} \Theta}{B+C \Omega^{k}}+D \Theta
$$

Taking the difference

$$
\Omega-\Theta=\frac{\sum_{i=1}^{l} A_{s_{i}} \Omega}{B+C \Theta^{k}}-\frac{\sum_{i=1}^{l} A_{s_{i}} \Theta}{B+C \Omega^{k}}+D(\Omega-\Theta),
$$

we deduce that

$$
(1-D)(\Omega-\Theta)-\frac{\sum_{i=1}^{l} A_{s_{i}} \Omega\left(B+C \Omega^{k}\right)-\sum_{i=1}^{l} A_{s_{i}} \Theta\left(B+C \Theta^{k}\right)}{\left(B+C \Theta^{k}\right)\left(B+C \Omega^{k}\right)}=0,
$$

which implies

$$
\left[(1-D)-\frac{\sum_{i=1}^{l} A_{s_{i}} B+C \sum_{i=1}^{l} A_{s_{i}} \sum_{p+q=k} \Omega^{p} \Theta^{q}}{\left(B+C \Theta^{k}\right)\left(B+C \Omega^{k}\right)}\right](\Omega-\Theta)=0,
$$

where $\sum_{p+q=k} \Omega^{p} \Theta^{q}=\Omega^{k}+\Omega^{k-1} \Theta+\cdots+\Omega \Theta^{k-1}+\Theta^{k}$. 
By view of $0<D<1, \sum_{i=1}^{l} A_{s_{i}}<B^{2}(1-D) /((k+1) C+B)$ and initial conditions $\left(x_{-m}, x_{-m+1}, \ldots, x_{1}, x_{0}\right) \in(0,1)^{m+1}$, therefore we have

$$
(1-D)-\frac{\sum_{i=1}^{l} A_{s_{i}} B+C \sum_{i=1}^{l} A_{s_{i}} \sum_{p+q=k} \Omega^{p} \Theta^{q}}{\left(B+C \Theta^{k}\right)\left(B+C \Omega^{k}\right)}>(1-D)-\frac{[B+C(k+1)] \sum_{i=1}^{l} A_{s_{i}}}{B^{2}}>0,
$$

thus, we have

$$
\Omega=\Theta
$$

By Theorem 3.2, then every solution of (1.4) converges to the unique equilibrium point $\bar{x}=$ 0 .

Theorem 3.4. If $0<D<1, \sum_{i=1}^{l} A_{s_{i}}<B^{2}(1-D) /((k+1) C+B), s_{1} \neq \cdots \neq s_{l} \neq t_{1} \neq \cdots \neq t_{k}$, and $t_{j} \neq 0(j \in\{1,2, \ldots, k\})$, then the unique equilibrium point $\bar{x}=0$ of (1.4) is globally asymptotically stable for any initial conditions $\left(x_{-m}, x_{-m+1}, \ldots, x_{1}, x_{0}\right) \in(0,1)^{m+1}$.

Proof. The result holds from Theorems 3.1 and 3.3.

Theorem 3.5. If $0<D<1, \sum_{i=1}^{l} A_{s_{i}}<B^{2}(1-D) /\left((k+1) \mu^{k} C+B\right)$, and $t_{j} \neq 0(j \in\{1,2, \ldots, k\})$, then the unique equilibrium point $\bar{x}=0$ of (1.4) is global attractor for any initial conditions $\left(x_{-m}, x_{-m+1}, \ldots, x_{1}, x_{0}\right) \in(0, \mu)^{m+1}$.

Proof. The proof of this Theorem is similar to that of Theorem 3.3. We omit the details.

Theorem 3.6. If $0<D<1, \sum_{i=1}^{l} A_{s_{i}}<B^{2}(1-D) /\left((k+1) \mu^{k} C+B\right), s_{1} \neq \cdots \neq s_{l} \neq t_{1} \neq \cdots \neq t_{k}$, and $t_{j} \neq 0(j \in\{1,2, \ldots, k\})$, then the unique equilibrium point $\bar{x}=0$ of (1.4) is globally asymptotically stable for any initial conditions $\left(x_{-m}, x_{-m+1}, \ldots, x_{1}, x_{0}\right) \in(0, \mu)^{m+1}$.

Proof. The result holds from Theorems 3.1 and 3.5.

\section{Numerical Simulation}

In this section, we give some numerical simulations supporting our theoretical analysis via the software package Matlab 7.1. As examples, we consider

$$
\begin{gathered}
x_{n+1}=\frac{0.05 x_{n-2}+0.02 x_{n-4}+0.03 x_{n}}{8+x_{n-1} x_{n-3}}+\frac{1}{2} x_{n} \\
x_{n+1}=\frac{0.3 x_{n-4}+0.2 x_{n-3}}{10+3 x_{n-1} x_{n-2}}+\frac{1}{2} x_{n} \\
x_{n+1}=\frac{x_{n-4}+x_{n-3}}{8+3 x_{n-1} x_{n-2}}+2 x_{n} .
\end{gathered}
$$




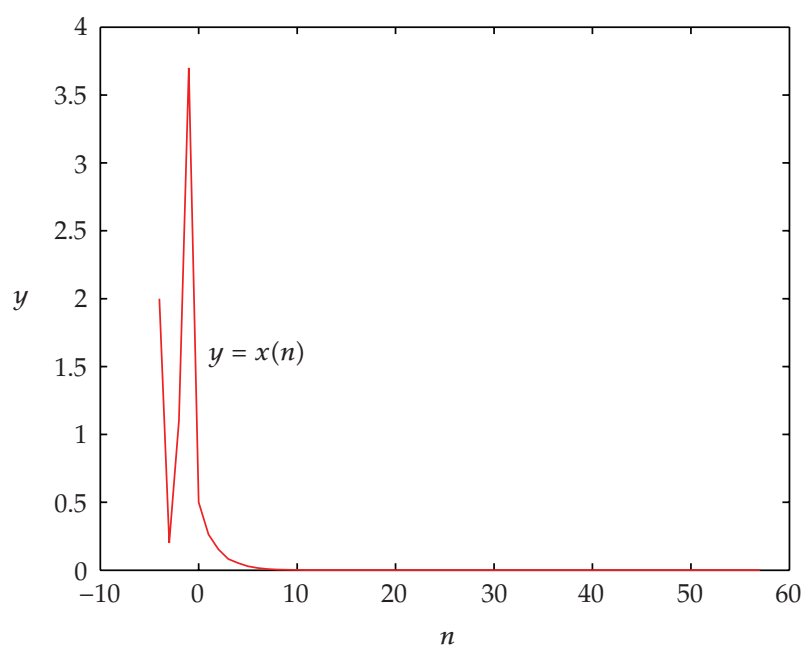

Figure 1

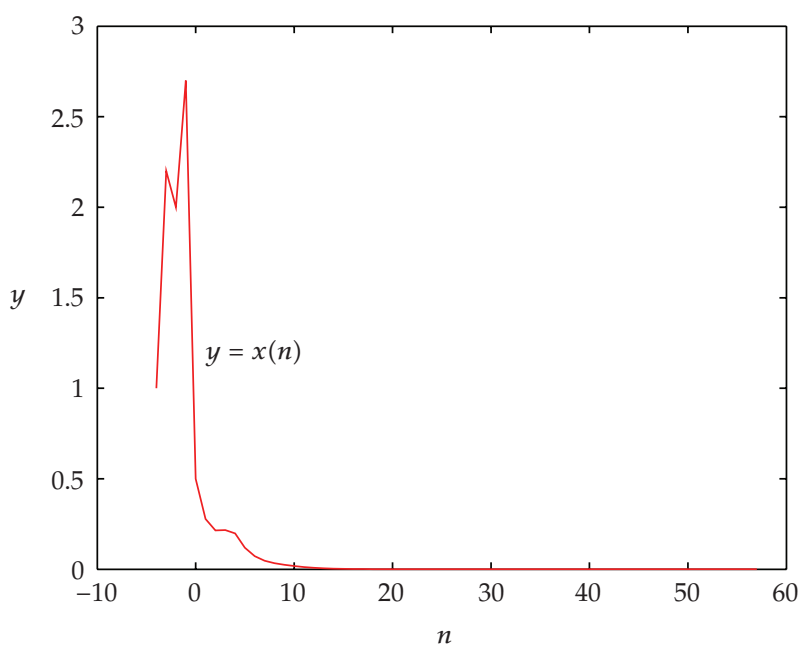

Figure 2

Let $\mu=4$, it is obvious that (4.1) and (4.2) satisfy the conditions of Theorem 3.6 when the initial datas $\left(x_{-4}, x_{-3}, x_{-2}, x_{-1}, x_{0}\right) \in(0,4)^{5}$. Equation (4.3) satisfies the conditions of Theorem 3.1 for the initial data $x_{-4}, x_{-3}, x_{-2}, x_{-1}, x_{0} \in \mathbb{R}^{+}$.

By employing the software package Matlab 7.1, we can solve the numerical solutions of (4.1), (4.2), and (4.3) which are shown, respectively, in Figures 1, 2, and 3. More precisely, Figure 1 shows the asymptotic behavior of the solution to (4.1) with initial data $x_{-4}=2, x_{-3}=$ $0.2, x_{-2}=1.1, x_{-1}=3.7$, and $x_{0}=0.5$, Figure 2 shows the asymptotic behavior of the solution to (4.2) with initial data $x_{-4}=1, x_{-3}=2.2, x_{-2}=2, x_{-1}=2.7$, and $x_{0}=0.5$, and Figure 3 shows the asymptotic behavior of the solution to (4.3) with initial data $x_{-4}=0.1, x_{-3}=$ 2.7, $x_{-2}=1.1, x_{-1}=3.7$, and $x_{0}=0.5$. 


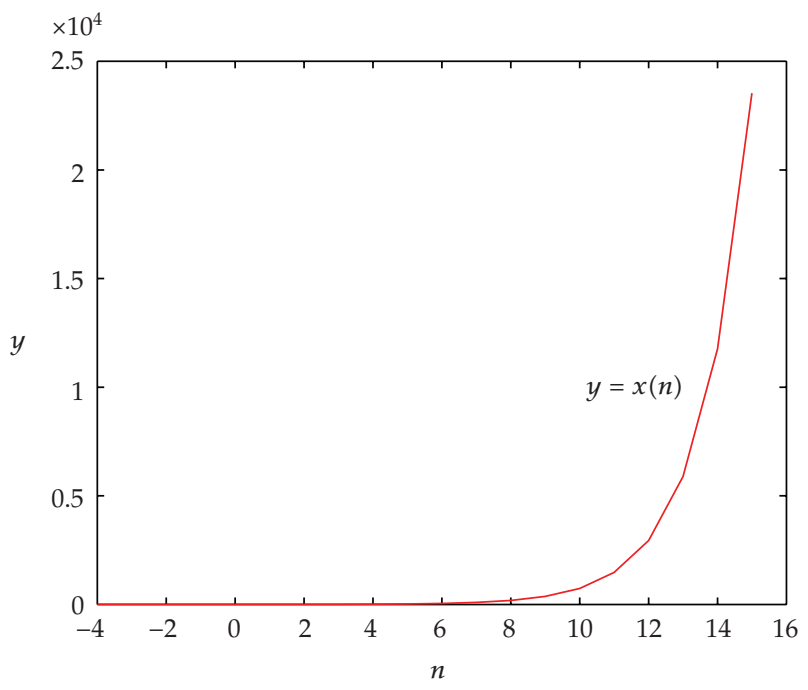

Figure 3

\section{Conclusions}

This paper presents the use of a variational iteration method for systems of nonlinear difference equations. This technique is a powerful tool for solving various difference equations and can also be applied to other nonlinear differential equations in mathematical physics. The numerical simulations show that this method is an effective and convenient one. The variational iteration method provides an efficient method to handle the nonlinear structure. Computations are performed using the software package Matlab 7.1.

We have dealt with the problem of global asymptotic stability analysis for a class of nonlinear difference equation. The general sufficient conditions have been obtained to ensure the existence, uniqueness, and global asymptotic stability of the equilibrium point for the nonlinear difference equation. These criteria generalize and improve some known results. In particular, some examples are given to show the effectiveness of the obtained results. In addition, the sufficient conditions that we obtained are very simple, which provide flexibility for the application and analysis of nonlinear difference equation.

\section{Acknowledgments}

The authors are grateful to the referee for giving us lots of precious comments. This work is supported by Natural Science Foundation Project of CQ CSTC (Grant no. 2008BB 7415) of China, and National Science Foundation (Grant no. 40801214) of China.

\section{References}

[1] W.-T. Li and H.-R. Sun, "Global attractivity in a rational recursive sequence," Dynamic Systems and Applications, vol. 11, no. 3, pp. 339-345, 2002.

[2] V. L. Kocić and G. Ladas, Global Behavior of Nonlinear Difference Equations of Higher Order with Applications, vol. 256 of Mathematics and Its Applications, Kluwer Academic Publishers, Dordrecht, The Netherlands, 1993. 
[3] M. R. S. Kulenović and G. Ladas, Dynamics of Second Order Rational Difference Equations with Open Problems and Conjectures, Chapman \& Hall/CRC, Boca Raton, Fla, USA, 2002.

[4] H. M. El-Owaidy, A. M. Ahmed, and M. S. Mousa, “On the recursive sequences $x_{n+1}=-\alpha x_{n-1} /(\beta \pm$ $\left.x_{n}\right), "$ Applied Mathematics and Computation, vol. 145, no. 2-3, pp. 747-753, 2003.

[5] X.-Y. Xianyi and D.-M. Zhu, "Global asymptotic stability in a rational equation," Journal of Difference Equations and Applications, vol. 9, no. 9, pp. 833-839, 2003.

[6] C. Çinar, "On the positive solutions of the difference equation $x_{n+1}=x_{n-1} /\left(1+x_{n} x_{n-1}\right)$," Applied Mathematics and Computation, vol. 150, no. 1, pp. 21-24, 2004.

[7] X. Yang, W. Su, B. Chen, G. M. Megson, and D. J. Evans, "On the recursive sequence $x_{n}=\left(a x_{n-1}+\right.$ $\left.b x_{n-2}\right) /\left(c+d x_{n-1} x_{n-2}\right), "$ Applied Mathematics and Computation, vol. 162, no. 3, pp. 1485-1497, 2005.

[8] K. S. Berenhaut, J. D. Foley, and S. Stević, "The global attractivity of the rational difference equation $y_{n}=\left(y_{n-k}+y_{n-m}\right) /\left(1+y_{n-k} y_{n-m}\right), "$ Applied Mathematics Letters, vol. 20, no. 1, pp. 54-58, 2007.

[9] K. S. Berenhaut and S. Stević, "The difference equation $x_{n+1}=a+x_{n-k} / \sum_{i=0}^{k-1} c_{i} x_{n-i}$ has solutions converging to zero," Journal of Mathematical Analysis and Applications, vol. 326, no. 2, pp. 1466-1471, 2007.

[10] R. Memarbashi, "Sufficient conditions for the exponential stability of nonautonomous difference equations," Applied Mathematics Letters, vol. 21, no. 3, pp. 232-235, 2008.

[11] M. Aloqeili, "Global stability of a rational symmetric difference equation," Applied Mathematics and Computation, vol. 215, no. 3, pp. 950-953, 2009.

[12] M. Aprahamian, D. Souroujon, and S. Tersian, "Decreasing and fast solutions for a second-order difference equation related to Fisher-Kolmogorov's equation," Journal of Mathematical Analysis and Applications, vol. 363, no. 1, pp. 97-110, 2010.

[13] C.-y. Wang, F. Gong, S. Wang, L.-r. Li, and Q.-h. Shi, "Asymptotic behavior of equilibrium point for a class of nonlinear difference equation," Advances in Difference Equations, vol. 2009, Article ID 214309, 8 pages, 2009.

[14] C.-y. Wang, S. Wang, Z.-w. Wang, F. Gong, and R.-f. Wang, "Asymptotic stability for a class of nonlinear difference equations," Discrete Dynamics in Nature and Society, vol. 2010, Article ID 791610, 10 pages, 2010. 